\title{
Ileal non-Hodgkin's lymphoma presenting as perforation
}

\author{
Sanjay Kumar Yadav • Jitin Yadav • Abhinav Anand • \\ Rajeev Ranjan Kumar
}

Accepted: 1 February 2015 / Published online: 15 February 2015

(C) Springer-Verlag Berlin Heidelberg 2015

Dear Editor,

We read the article entitled "Intestinal lymphoma-a review of the management of emergency presentations to the general surgeon" by S. Abbott et al. [1] In the International Journal Of Colorectal Diseases (Feb. 2015). The authors are right that there is a lack of quality evidence for the elective and emergency treatment of non-Hodgkin's lymphoma (NHL) involving the small and large intestine. There is a lack of information especially to general surgeons regarding management of intestinal lymphoma presenting as an emergency. We encountered a 15 -year-old male patient who presented to us with features of perforation peritonitis. He was taken up for emergency exploratory laparotomy. We noticed a firm mass approximately $15 \times 15 \mathrm{~cm}$ in the mid ileum with a perforation. The mass was resected out, and end to end ileoileal anastomosis was done. Final histopathology of the mass came as T cell NHL. Patient was put up on chemotherapy CVP regimen (cyclophosphamide $750 \mathrm{mg} / \mathrm{m}^{2}$ iv day 1 , vincristine $1.4 \mathrm{mg} / \mathrm{m}^{2}$ iv day 1, prednisone $40 \mathrm{mg} / \mathrm{m}^{2}$ per oral qd days 1-5 Q3 weeks $\times$ 8 cycles). Patient responded well and is currently in follow-up after 1 year of completion of chemotherapy. We agree with the authors' comments that in order to develop evidence-based treatment protocols, there should be an intestinal NHL registry.

Conflict of interest Authors declare no conflict of interest.

Funding No external funding source was provided.

\section{References}

1. Abbott S, Nikolousis E, Badger I (2015) Intestinal lymphoma - a review of the management of emergency presentations to the general surgeon. Int J Colorectal Dis 30(2):151-157. doi:10.1007/s00384014-2061-1

Electronic supplementary material The online version of this article (doi:10.1007/s00384-015-2151-8) contains supplementary material, which is available to authorized users.

S. K. Yadav $(\bowtie) \cdot J$. Yadav $\cdot$ A. Anand $\cdot$ R. R. Kumar Department of General Surgery, Rajendra Institute of Medical Sciences, Hostel No. 03, Room No. 50, Rims,

Ranchi, Jahrkhand 834009, India

e-mail: sky1508@gmail.com 